\title{
Osimertinib alone as second-line treatment for brain metastases (BM) control may be more limited than for non-BM in advanced NSCLC patients with an acquired EGFR T790M mutation
}

\author{
Changhui $\mathrm{Li}^{1+}$, Wei Nie ${ }^{1 \dagger}$, Jingdong Guo ${ }^{2 \dagger}$, Anning Xiong ${ }^{1 \dagger}$, Hua Zhong ${ }^{1}$, Tianqing Chu', Runbo Zhong ${ }^{1}$, \\ Jianlin Xu', Jun Lu' ${ }^{1}$ Xiaoxuan Zheng ${ }^{1}$, Bo Zhang ${ }^{1}$, Yinchen Shen ${ }^{1}$, Feng Pan ${ }^{1}$, Baohui Han ${ }^{1 *}$ and \\ Xueyan Zhang ${ }^{1 *}$ (D)
}

\begin{abstract}
Background: This study was designed to investigate the difference between brain metastases (BM) and non-brain metastases (non-BM) treated by osimertinib in advanced patients with an acquired EGFRT790M mutation after obtaining first-generation EGFR-TKI resistance.

Methods: A total number of 135 first-generation EGFR-TKI-resistant patients with an acquired EGFR T790M mutation were retrospectively analyzed. The patients were divided into BM and non-BM groups. According to the type of treatment (whether brain radiotherapy), the BM patients were divided into an osimertinib combined with brain radiotherapy group and an osimertinib without brain radiotherapy group. In addition, according to the type of BM (the sequence between BM and osimertinib), the BM patients were subdivided into an osimertinib after BM group (initial BM developed after obtaining first-generation EGFR-TKI resistance) and an osimertinib before BM group (first-generation EGFR-TKI resistance then osimertinib administration performed; initial BM was not developed until osimertinib resistance). The progression-free survival (PFS) and overall survival (OS) were evaluated. The primary endpoint was OS between BM and no-BM patients. The secondary endpoints were PFS of osimertinib, and OS between brain radiotherapy and non-brain radiotherapy patients.
\end{abstract}

Results: A total of 135 patients were eligible and the median follow-up time of all patients was 50 months. The patients with BM $(n=54)$ had inferior OS than those without BM $(n=81)(45$ months vs. 55 months, $P=0.004)$. And in BM group, the OS was longer in patients that received osimertinib combined with brain radiotherapy than in those without brain radiotherapy (53 months vs. 40 months, $P=0.014$ ). In addition, the PFS was analysed according to whether developed BM after osimertinib resistance. The PFS of the patients that developed BM after acquiring osimertinib resistance was shorter than that without BM development, whether patients developed initial BM after

\footnotetext{
*Correspondence: xkyyhan@gmail.com; zxychest@163.com

${ }^{\dagger}$ Changhui Li, Wei Nie, Jingdong Guo and Anning Xiong contributed equally to this work

1 Department of Pulmonary, Shanghai Chest Hospital, Shanghai Jiao Tong

University, No.241 Huaihai West Road, Xuhui District, Shanghai 200030,

China

Full list of author information is available at the end of the article
}

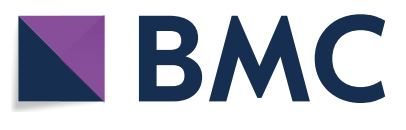

(c) The Author(s) 2021. Open Access This article is licensed under a Creative Commons Attribution 4.0 International License, which permits use, sharing, adaptation, distribution and reproduction in any medium or format, as long as you give appropriate credit to the original author(s) and the source, provide a link to the Creative Commons licence, and indicate if changes were made. The images or other third party material in this article are included in the article's Creative Commons licence, unless indicated otherwise in a credit line to the material. If material is not included in the article's Creative Commons licence and your intended use is not permitted by statutory regulation or exceeds the permitted use, you will need to obtain permission directly from the copyright holder. To view a copy of this licence, visit http://creativecommons.org/licenses/by/4.0/. The Creative Commons Public Domain Dedication waiver (http://creativeco mmons.org/publicdomain/zero/1.0/) applies to the data made available in this article, unless otherwise stated in a credit line to the data. 
first-generation EGFR-TKI resistance (7 months vs. 13 months, $P=0.003$ ), or developed non-BM after first-generation EGFR-TKI resistance (13 months vs. 17 months, $P<0.001)$.

Conclusions: In advanced patients with an acquired EGFR T790M mutation after obtaining first-generation EGFRTKI resistance, osimertinib may be more limited in its control in BM than in non-BM. Also, osimertinib combined with brain radiotherapy may improve the survival time of BM patients.

Keywords: Osimertinib, Brain metastases, EGFRT790M mutation, Radiotherapy

\section{Background}

Epidermal growth factor receptor (EGFR)-tyrosine kinase inhibitor (TKI) significantly improves the survival time of advanced non-small cell lung cancer (NSCLC) patients with sensitive EGFR mutation $[1,2]$. However, in most patients, the disease progresses after administration of the first-generation of EGFR-TKI for 10-13 months [3]. Due to the presence of a blood-brain barrier (BBB), first-generation EGFR-TKI exerts poor control in the brain [4]. Thus, brain metastasis (BM) is one of the common accounting for $40 \%$ of the first-generation EGFR-TKI-resistant metastasis sites [5, 6]. No effective treatment is available once BM has developed, which significantly shortens patient survival time [7]. Therefore, it is critically important to prolong the survival time of BM patients with an acquired EGFR T790M mutation.

The emergence of osimertinib has obviously improved the survival time in BM patients with an EGFR-sensitive mutation and an acquired EGFR T790M mutation [8]. However, due to the high price of osimertinib, it is not cost-effective to use osimertinib as the first-line treatment even in developed countries such as Canada [9]. In developing countries such as China, osimertinib is used mainly for the treatment of patients, especially with BM, with an acquired T790M mutation after obtaining first-generation EGFR-TKI resistance. However, it is still unclear whether osimertinib exerts different effects in advanced BM and non-BM patients with an acquired EGFR T790M mutation after the occurrence of firstgeneration EGFR-TKI resistance. Furthermore, in the first-generation EGFR-TKI-resistant patients, even if BM did not occur before the administration of osimertinib, it may develop after osimertinib resistance. Nonetheless, it is still not elucidated whether there is a difference in the survival between patients with BM developed before osimertinib administration and those with BM developed after osimertinib resistance.

A previous study showed that first-generation EGFRTKI combined with radiotherapy (RT) improved significantly more the survival time of BM patients than EGFR-TKI alone [10]. Nevertheless, whether osimertinib combined with other treatments can further prolong survival time is also worth discussing. Further research is required to establish whether osimertinib combined with
RT could also bring survival benefits for BM patients with an acquired T790M mutation.

Therefore, this retrospective study was conducted to investigate whether there is a difference between BM and non-BM in the effects of treatment with osimertinib in advanced patients with an acquired EGFR T790M mutation after obtaining first-generation EGFR-TKI resistance. We have also discussed the survival difference between patients with initial BM developed before osimertinib administration and patients with initial BM developed after acquiring osimertinib resistance. In addition, we investigated whether osimertinib combined with brain RT could bring additional survival benefits to the aforementioned BM patients.

\section{Materials and methods Patient selection}

From June 1, 2016 to December 31, 2018, a total number of 1180 advanced patients with (EGFR)-mutant lung adenocarcinoma were screened in Shanghai Chest Hospital (Shanghai Chest Hospital, Shanghai Jiao Tong University, PR China). Finally, 135 patients who met the inclusion criteria were enrolled in the study.

The selection criteria were as follows: (1) Patients with stage IV NSCLC; (2) Histological or cytological examinations showed adenocarcinoma, and the gene detection was EGFR-sensitive mutation before initial treatment (19 deletion or 21 L858R mutation); (3) No BM at the initial diagnosis; (4) The first-generation EGFR-TKI was used as first-line treatment; (5) Acquired T790M mutation was detected after obtaining first-generation EGFR-TKI resistance, and osimertinib was applied as a second-line treatment.

The following exclusion criteria were employed: (1) The patients had a resistance mutation or an invalid EGFR mutation; (2) Non-T790M mutation after obtaining firstgeneration EGFR-TKI resistance; (3) BM developed but not as first-generation EGFR-TKI or osimertinib resistance, or meningeal metastasis developed; (4) Osimertinib was not administered during the treatment or was applied as the first-line treatment; (5) The first-generation EGFR-TKI or osimertinib was combined with other chemotherapy. 
The following examinations were used as a clinical baseline assessment for all patients: chest computed tomography (CT), brain magnetic resonance imaging (MRI), bone scan, and abdominal ultrasound. After 4 weeks of treatment with the first-generation EGFR-TKI or osimertinib, the patients were subjected to chest CT and abdominal ultrasound. Then, they underwent chest CT and abdominal ultrasound every 3-4 months in the follow-up treatment until progression occurred. Furthermore, bone scan was conducted every 4-6 months, and brain MRI was done every 3 months. If the patient developed brain symptoms during the treatment, brain MRI was immediately performed to confirm the diagnosis. In addition, the BM patients received MRI every 2-3 months.

\section{Study design}

Patients' medical records and follow-up data were collected. The following information was recorded: age, sex, smoking history, EGFR mutation type, Eastern Cooperative Oncology Group (ECOG) performance status (PS), time of BM development, BM symptoms, diameter of the largest BM, number of BM, and information on received brain RT or not and RT type. Depending on the BM stage, disease-specific graded prognosis assessment (DS-GPA) was also conducted [11, 12]. The baseline data of patients were classified according to the following criteria: age $(<60$ or $\geq 60)$, sex (male or female), smoking history (yes or no), EGFR mutation (19 deletion or 21 L858R), ECOG-PS (0-1 or 2-3), the largest size of $\mathrm{BM}(<1$ or $\geq 1 \mathrm{~cm})$, the number of $\mathrm{BM}(\leq 3$ or $>3)$, BM symptoms(yes or no), DS-GPA score (0-1.5 or 2-4), and information on received brain RT or not and RT type [none, whole-brain radiation therapy (WBRT) or stereotactic radiosurgery (SRS)].

The patients were divided into two groups: patients with BM $(n=54)$ and patients without BM $(n=81)$. According to the type of treatment (whether brain radiotherapy), the BM patients were divided into osimertinib combined with brain radiotherapy group $(n=37)$ and osimertinib without brain radiotherapy group $(n=17)$. In addition, according to the type of $\mathrm{BM}$ (the sequence between BM and osimertinib), the BM patients were subdivided into an osimertinib after BM group (initial BM developed after obtaining first-generation EGFR-TKI resistance, and then taking osimertinib) $(n=33)$ and an osimertinib before BM group (first-generation EGFR-TKI resistance obtained followed by osimertinib intake and initial BM was not developed until osimertinib resistance had occurred) $(n=21)$. The study has been approved by the Ethics Committee and the Institutional Review Board, and was carried out in accordance with Helsinki
Declaration. An informed consent form was signed by each patient before data collection.

\section{Evaluation criteria and treatment options}

The extracranial lesions were examined and evaluated by chest $\mathrm{CT}$, bone scan, and abdominal ultrasound. The intracranial lesions were assessed by brain MRI. If biopsy could not be performed after obtaining first-generation EGFR-TKI resistance, liquid biopsy (plasma-circulating tumor DNA [ctDNA]) was done. The EGFR mutation detection kit (Amoy Diagnostics, Xiamen, China) was used, based on mutation amplification system technology (ARMS), to detect the most common types of EGFR mutations. The oral doses of the first-generation EGFRTKI were $150 \mathrm{mg}$ (erlotinib) per day, $250 \mathrm{mg}$ (gefitinib) per day, or $125 \mathrm{mg}$ (icotinib) three times per day. The oral dose of osimertinib was $80 \mathrm{mg}$ per day.

Progression-free survival (PFS) was defined as the time interval between the initiation time of osimertinib administration and the time of osimertinib resistance development. In addition, overall survival (OS) was defined as the time interval from the start of the firstgeneration EGFR-TKIs application to the death (July 1, 2020). And for patients alive at the data cutoff date, OS was censored at the last follow-up date. The primary endpoint was OS between BM and no-BM patients. The secondary endpoints were PFS of osimertinib, and OS between brain radiotherapy and non-brain radiotherapy patients.

\section{Data analysis}

The categorical variables of patient characteristics were analyzed by $x^{2}$ test. PFS and OS were assessed by the Kaplan-Meier method, and further comparison was performed using the log rank test. Finally, the Cox proportional hazards regression model was applied for multivariate analysis to determine the independent prognostic factors related to OS. A P-value less than 0.05 was considered to indicate a statistically significant difference.

All statistics were analyzed using SPSS software version 23.0 (IBM Corporation, NY, USA), and the Prism software was used for plotting survival curves in this study.

\section{Results}

\section{Patient characteristics}

From June 1, 2016 to December 31, 2018, a total number of 1180 advanced patients with EGFR mutation lung adenocarcinoma were screened. Of them, 1045 patients were excluded due to failure to meet the inclusion criteria (Resistance mutation or invalid EGFR mutation $(n=84)$; No T790M mutation after first-generation EGFR-TKI resistance $(n=256) ; 248$ patients developed meningeal metastasis, or developed BM but did not appear during 
first-generation EGFR-TKI or osimertinib treatment; 347 patients was not administered osimertinib during the treatment or was applied as the first-line treatment; and 110 patients received the first-generation EGFR-TKI or osimertinib combined with other chemotherapy). Finally, 135 advanced patients, who were EGFR T790M mutation-positive after obtaining first-generation EGFR-TKI resistance and treated by osimertinib as the second-line treatment, met the inclusion criteria and were enrolled for analysis. The flow chart of patient screening is presented in Fig. 1.

Of these 135 patients, 81 (60\%) did not develop BM during the entire course of treatment, whereas 54 (40\%) developed BM after first-generation EGFR-TKI or osimertinib resistance. Of the BM patients group, 33 patients $(24.4 \%)$ took osimertinib after initial BM development (BM initial developed after obtaining firstgeneration EGFR-TKI resistance, followed by osimertinib administration), and 21 (15.6\%) developed BM after acquiring osimertinib resistance (first-generation EGFR-TKI resistance occurred, and then osimertinib was administered; initial BM had not been developed until osimertinib resistance had occurred).

The median follow-up time for these 135 patients was 50 months (95\% CI 43.45-56.55). The median ages in the $\mathrm{BM}$ and non-BM groups were 62 years (36-83 years) and 63 years (35-84 years), respectively. The baseline characteristics of the patients are listed in Table 1. No differences were found in age, sex, smoking history, EGFR mutation and ECOG-PS between the BM group and the non-BM group.

\section{Survival analysis of OS in all patients}

On July 1, 2020, which was considered the cut-off point, $50 \%(n=68)$ of the patients were still alive (21 cases with $\mathrm{BM}$ progression and 47 cases without $\mathrm{BM}$ progression). The remaining 67 patients (50\%) died during the followup period. The median follow-up time in all patients was 50 months; the patients without $\mathrm{BM}$ during the treatment had a longer OS than those with BM (55 months vs. 45 months, $P=0.004$ ) (Fig. 2a).

After controlling important covariates in the multivariable model, sex and whether developed BM were independently related to OS among all patients (Fig. 2b). Female had significantly longer OS than male (HR 0.262; CI 0.131 to $0.521 ; \mathrm{P}<0.001)$. And patients with $\mathrm{BM}$ had significantly shorter OS than those without BM (HR 0.558 ; CI 0.340 to 0.917 ; $\mathrm{P}=0.021$ ).

\section{Survival analysis of OS in BM patients}

The baseline characteristics of the BM patients are presented in Table 2. No differences were established in age, sex, smoking history, ECOG-PS, EGFR mutation, BMrelated characteristics, DS-GPA, and BM RT between the osimertinib after BM group and the osimertinib before BM group.

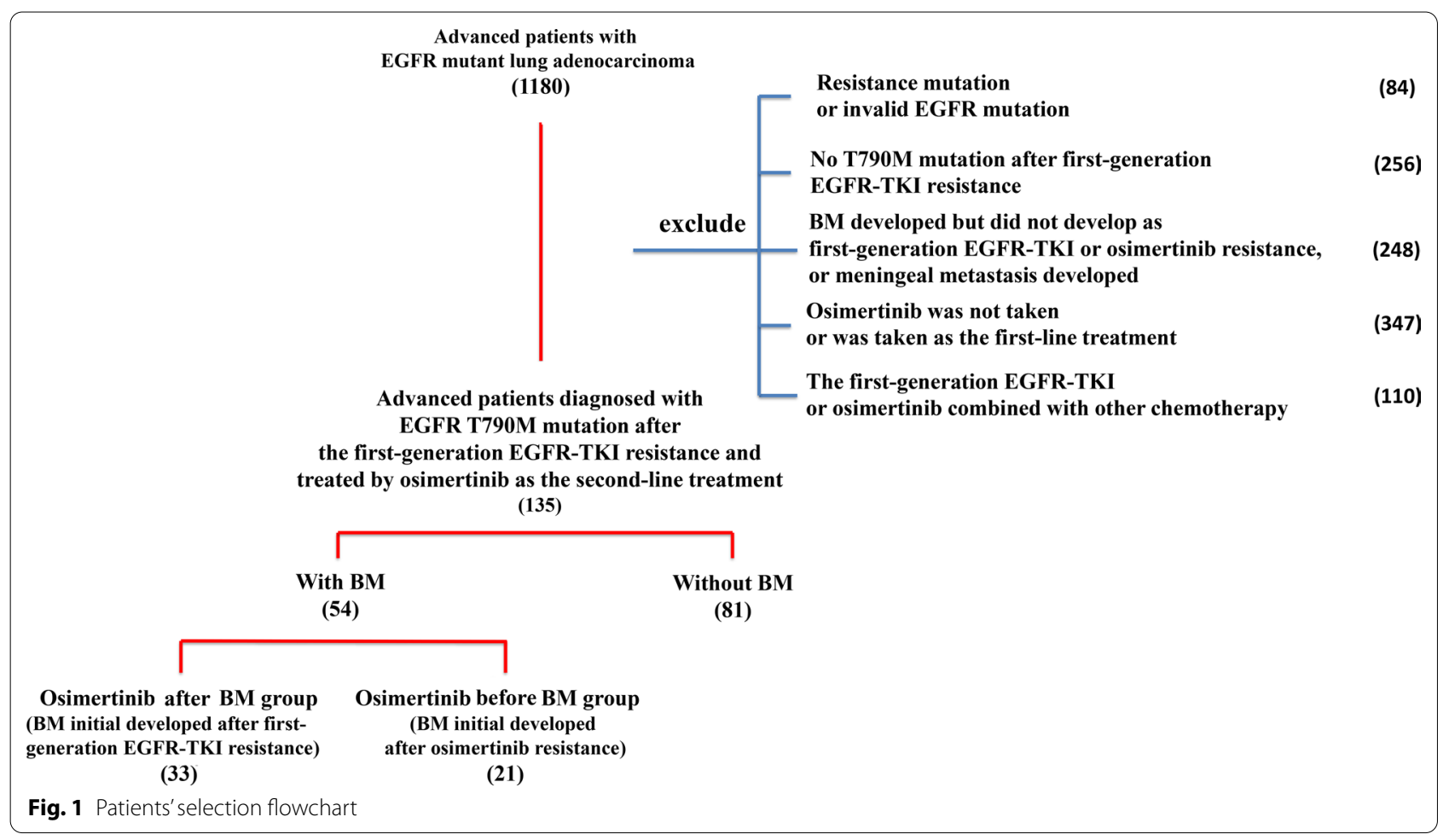


Table 1 Patients characteristics

\begin{tabular}{|c|c|c|c|c|}
\hline Characteristics & $\begin{array}{l}\text { BM group } \\
\text { No. }(\%)\end{array}$ & $\begin{array}{l}\text { Non-BM group } \\
\text { No. (\%) }\end{array}$ & $\mathbf{P}$ & $\begin{array}{l}\text { Total } \\
\text { No. (\%) }\end{array}$ \\
\hline \multicolumn{5}{|l|}{ Age } \\
\hline$<60$ & $20(14.8 \%)$ & $22(16.3 \%)$ & 0.225 & $42(31.1 \%)$ \\
\hline$\geq 60$ & $34(25.2 \%)$ & $59(43.7 \%)$ & & $93(68.9 \%)$ \\
\hline \multicolumn{5}{|l|}{ Sex } \\
\hline Male & $26(19.3 \%)$ & $32(23.7 \%)$ & 0.320 & $58(43.0 \%)$ \\
\hline Female & $28(20.7 \%)$ & $49(36.3 \%)$ & & $77(57.0 \%)$ \\
\hline \multicolumn{5}{|l|}{ Smoking history } \\
\hline Yes & $18(13.4 \%)$ & $28(20.7 \%)$ & 0.882 & $46(34.1 \%)$ \\
\hline No & $36(26.7 \%)$ & $53(39.2 \%)$ & & $89(65.9 \%)$ \\
\hline \multicolumn{5}{|l|}{ EGFR mutation } \\
\hline 19 del & $30(22.2 \%)$ & $53(39.3 \%)$ & 0.248 & $83(61.5 \%)$ \\
\hline 21 L858R & $24(17.8 \%)$ & $28(20.7 \%)$ & & $52(38.5 \%)$ \\
\hline \multicolumn{5}{|l|}{ ECOG-PS } \\
\hline $0-1$ & $48(35.6 \%)$ & 75 (55.6\%) & 0.459 & $123(91.2 \%)$ \\
\hline $2-3$ & $6(4.4 \%)$ & $6(4.4 \%)$ & & $12(8.8 \%)$ \\
\hline \multicolumn{5}{|l|}{ Type of BM ${ }^{a}$} \\
\hline $\begin{array}{l}\text { Osimertinib after } \\
\text { BM }\end{array}$ & $33(61.1 \%)$ & - & & $33(61.1 \%)$ \\
\hline $\begin{array}{l}\text { Osimertinib before } \\
\text { BM }\end{array}$ & $21(38.9 \%)$ & - & & $21(38.9 \%)$ \\
\hline \multicolumn{5}{|c|}{ Size of the largest BM $(\mathrm{cm})^{\mathrm{a}}$} \\
\hline$<1$ & $38(70.4 \%)$ & - & & $38(70.4 \%)$ \\
\hline$\geq 1$ & $16(29.6 \%)$ & - & & $16(29.6 \%)$ \\
\hline \multicolumn{5}{|l|}{ Number of $\mathrm{BM}^{\mathrm{a}}$} \\
\hline$\leq 3$ & $14(25.9 \%)$ & - & & $14(25.9 \%)$ \\
\hline$>3$ & $40(74.1 \%)$ & - & & $40(74.1 \%)$ \\
\hline \multicolumn{5}{|l|}{ Symptom of $\mathrm{BM}^{\mathrm{a}}$} \\
\hline Yes & $12(22.2 \%)$ & - & & $12(22.2 \%)$ \\
\hline No & $42(77.8 \%)$ & - & & $42(77.8 \%)$ \\
\hline \multicolumn{5}{|l|}{ DS-GPA ${ }^{a}$} \\
\hline $0-1.5$ & $15(27.8 \%)$ & - & & $15(27.8 \%)$ \\
\hline $2-4$ & $39(72.2 \%)$ & - & & $39(72.2 \%)$ \\
\hline \multicolumn{5}{|l|}{ Radiotherapy (BM) ${ }^{\mathrm{a}}$} \\
\hline WBRT/SRS & $37(68.5 \%)$ & - & & $37(68.5 \%)$ \\
\hline No & $17(31.5 \%)$ & - & & $17(31.5 \%)$ \\
\hline
\end{tabular}

$B M$ brain metastases, EGFR epidermal growth factor receptor, WBRT whole brain radiation therapy, SRS stereotactic radiosurgery, ECOG Eastern cooperative oncology group, PS performance status, DS-GPA disease specific graded prognostic assessment

a Only for patients with BM

The median follow-up time in the BM patients was 45 months, and the patients that developed initial BM after first-generation EGFR-TKI administration had inferior OS than those that developed initial BM after osimertinib resistance ( 40 months vs. 55 months, $P=0.021$ ) (Fig. 3a). The patients that received brain RT had a significantly longer OS than those without brain RT (53 months vs. 40 months, $P=0.014$ ) (Fig. $3 \mathrm{~b}$ ).
Furthermore, in the multivariable model among $\mathrm{BM}$ patient group, male was an independent prognostic factor for inferior OS (HR 0.337; CI 0.163 to 0.697 ; $\mathrm{P}=0.003)$. And brain RT was an independent prognostic factor for superior OS (HR 0.272; CI 0.123 to 0.598 ; $\mathrm{P}=0.001)$.

\section{Survival analysis of BM}

The PFS in patients with initial BM developed after obtaining first-generation EGFR-TKI resistance group was 9 months. Of them, the patients that repeatedly developed BM after acquiring osimertinib resistance had shorter PFS and OS than the patients without BM development after acquiring osimertinib resistance (PFS: 7 months vs. 13 months, $P=0.003$, Fig. 4a; OS: 34 months vs. 49 months, $P=0.024$, Fig. $4 \mathrm{~b})$.

The PFS in patients without BM development after obtaining first-generation EGFR-TKI resistance group was 15 months. And the PFS of patients developed initial BM after acquiring osimertinib resistance was shorter than that without BM development (PFS: 13 months vs. 17 months, $P<0.001$, Fig. 4c). However, the patients with initial BM developed after acquiring osimertinib resistance did not show significant OS advantage over the patients without BM development (OS: 55 months vs. 55 months, $P=0.497$, Fig. $4 d$ ).

In addition, classification analysis was performed according to whether there was BM developed after osimertinib resistance. In patients developed BM after acquiring osimertinib resistance group, the patients with initial BM after first-generation EGFR-TKI resistance had shorter PFS than those without BM (7 months vs. 13 months, $P=0.017$ ) (Fig. 4e). However, in patients developed non-BM after osimertinib resistance group, the patients with initial BM after obtaining first-generation EGFR-TKI resistance failed to show superior PFS than those without BM (13 months vs. 17 months, $P=0.087$ ) (Fig. 4f).

\section{Discussion}

In this study, we discuss the efficacy of osimertinib as a second-line treatment in patients, especially BM patients, with EGFR T790M-positive advanced NSCLC who experience disease progression with prior first-generation EGFR-TKI treatment. Finally, 135 patients met the enrollment criteria and were analyzed. Our findings showed that in advanced patients with EGFR T790M mutation after obtaining first-generation EGFR-TKI resistance, osimertinib alone as the second-line treatment to control $\mathrm{BM}$ may be more limited in its action than in non-BM patients. The efficacy of osimertinib for patients that had developed initial BM before osimertinib administration was more limited than patients that had developed initial 


\section{Entire patients}

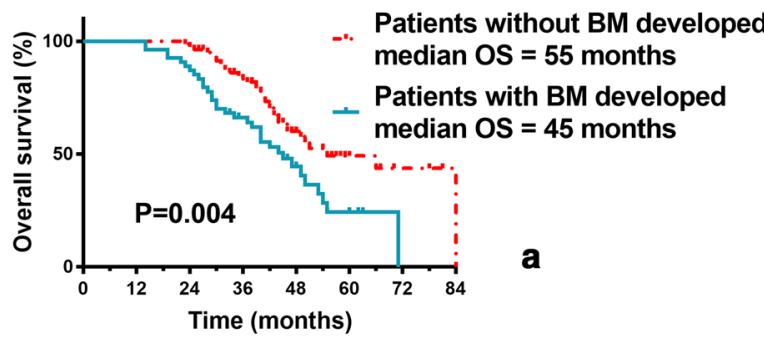

b

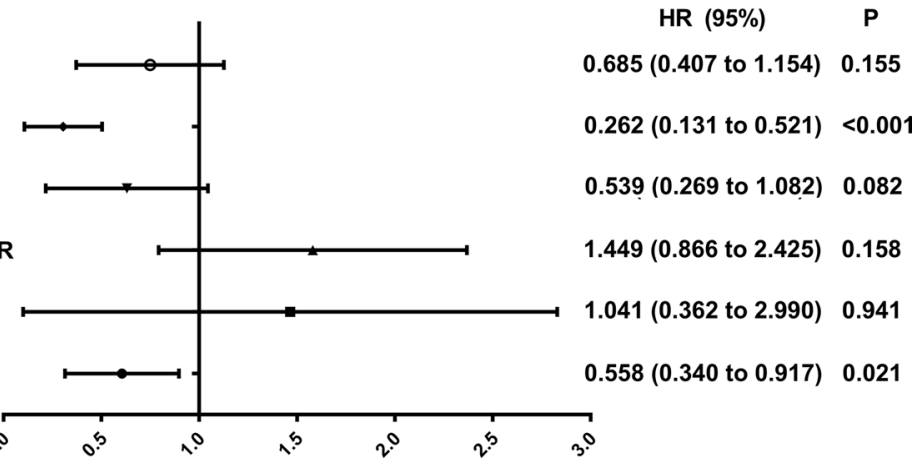

Fig. 2 Survival analysis of OS in all patients. a The patients without BM had a longer OS than those with BM; $\mathbf{b}$ In the multivariable model, sex and whether developed BM were independently related to OS among all patients
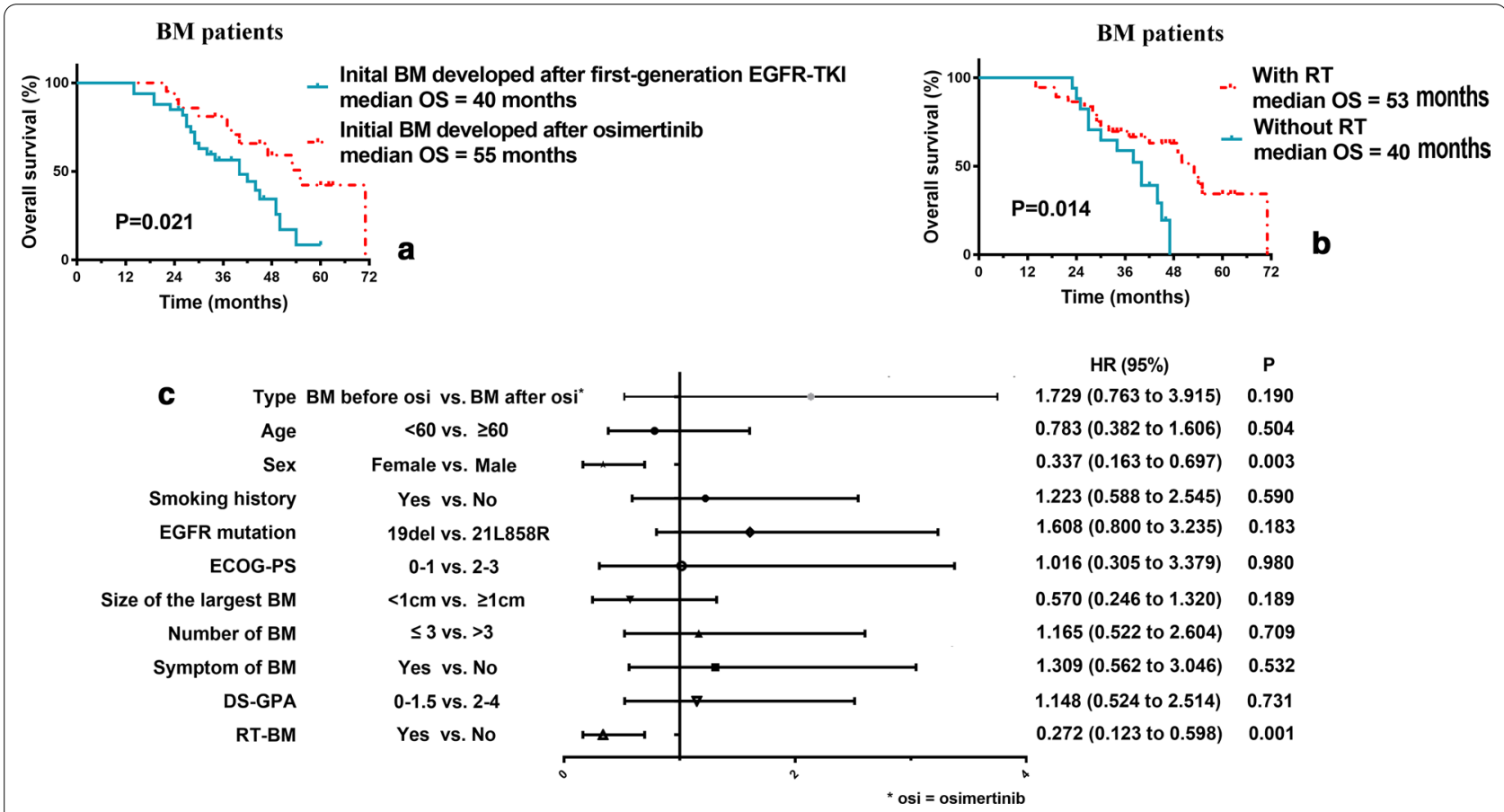

Fig. 3 Survival analysis of OS in BM patients. a The patients that developed initial BM after first-generation EGFR-TKIs had inferior OS than those that developed initial BM after osimertinib resistance had occurred; $\mathbf{b}$ The patients receiving RT had a significantly longer OS than those without RT; $\mathbf{c}$ In the multivariate model, sex, and brain RT were independently associated with OS in the BM patient group 
Table 2 Characteristics of patients with different types of BM

\begin{tabular}{|c|c|c|c|c|}
\hline \multirow[t]{2}{*}{ Characteristics } & \multirow{2}{*}{$\begin{array}{l}\text { Osimertinib after } \\
\text { BM } \\
\text { No. (\%) }\end{array}$} & \multirow{2}{*}{$\begin{array}{l}\text { Osimertinib } \\
\text { before BM } \\
\text { No. (\%) }\end{array}$} & \multirow[t]{2}{*}{$\mathbf{P}$} & \multirow{2}{*}{$\begin{array}{l}\text { Total } \\
\text { No. (\%) }\end{array}$} \\
\hline & & & & \\
\hline \multicolumn{5}{|l|}{ Age } \\
\hline$<60$ & $12(22.2 \%)$ & $8(14.8 \%)$ & 0.898 & $20(37.0 \%)$ \\
\hline$\geq 60$ & $21(38.9 \%)$ & $13(24.1 \%)$ & & $34(63.0 \%)$ \\
\hline \multicolumn{5}{|l|}{ Gender } \\
\hline Male & $18(33.3 \%)$ & $8(14.8 \%)$ & 0.238 & $26(48.1 \%)$ \\
\hline Female & $15(27.8 \%)$ & $13(24.1 \%)$ & & $28(51.9 \%)$ \\
\hline \multicolumn{5}{|c|}{ Smoking history } \\
\hline Yes & 13(24.1\%) & $5(9.3 \%)$ & 0.236 & $18(33.3 \%)$ \\
\hline No & $20(37.0 \%)$ & $16(29.6 \%)$ & & $36(66.7 \%)$ \\
\hline \multicolumn{5}{|l|}{ EGFR mutation } \\
\hline 19 del & $16(29.6 \%)$ & $14(25.9 \%)$ & 0.190 & $30(55.6 \%)$ \\
\hline 21 L858R & $17(31.5 \%)$ & $7(13.0 \%)$ & & $24(44.4 \%)$ \\
\hline \multicolumn{5}{|l|}{ ECOG-PS } \\
\hline $0-1$ & $29(53.7 \%)$ & 19 (35.2\%) & 0.767 & $48(88.9 \%)$ \\
\hline $2-3$ & $4(7.4 \%)$ & $2(3.7 \%)$ & & $6(11.1 \%)$ \\
\hline \multicolumn{5}{|c|}{ Size of the largest BM (cm) } \\
\hline$<1$ & $25(46.3)$ & $13(24.1)$ & 0.277 & $38(70.4 \%)$ \\
\hline$\geq 1$ & $8(14.8)$ & $8(14.8)$ & & $16(29.6 \%)$ \\
\hline \multicolumn{5}{|l|}{ Number of BM } \\
\hline$\leq 3$ & $9(16.7 \%)$ & $5(9.3 \%)$ & 0.777 & $14(26.0 \%)$ \\
\hline$>3$ & $24(44.4 \%)$ & $16(29.6 \%)$ & & $40(74.0 \%)$ \\
\hline \multicolumn{5}{|c|}{ Symptom of BM } \\
\hline Yes & $7(13.0 \%)$ & $5(9.3 \%)$ & 0.823 & $12(22.2 \%)$ \\
\hline No & $26(48.2 \%)$ & $16(29.6 \%)$ & & $42(77.8 \%)$ \\
\hline \multicolumn{5}{|l|}{ DS-GPA } \\
\hline $0-1.5$ & $8(14.8 \%)$ & $7(13.0 \%)$ & 0.467 & $15(27.8 \%)$ \\
\hline $2-4$ & $25(46.3 \%)$ & $14(25.9 \%)$ & & $39(72.2 \%)$ \\
\hline \multicolumn{5}{|c|}{ Radiotherapy (BM) } \\
\hline WBRT/SRS & $22(40.7 \%)$ & $15(27.8 \%)$ & 0.713 & $37(68.5 \%)$ \\
\hline No & $11(20.4 \%)$ & $6(11.1 \%)$ & & $17(31.5 \%)$ \\
\hline
\end{tabular}

$B M$ brain metastases, EGFR epidermal growth factor receptor, SRS stereotactic radiosurgery, WBRT whole brain radiation therapy, ECOG Eastern cooperative oncology group, PS performance status, DS-GPA disease specific graded prognostic assessment

$\mathrm{BM}$ after acquiring osimertinib resistance. It is noteworthy that osimertinib combined with brain RT may exert survival benefits in BM patients.

The AURA3 study found that osimertinib had better CNS efficacy in BM patients with an acquired T790M mutation after first-generation of EGFR-TKI resistance, but the treatment of the control group was pemetrexed combined with platinum [8]. The reason for the improved efficacy of osimertinib in BM patients may be the increased concentration of osimertinib in CSF as compared with those of previous drugs (such as first- and second-generation EGFR-TKI or chemotherapy) [13,
14]. However, whether there was a difference in the efficacy of osimertinib as second-line treatment between groups with $\mathrm{BM}$ and non-BM lesions is still unclear. Our research revealed that in advanced patients with an acquired EGFR T790M mutation after obtaining firstgeneration EGFR-TKI resistance, the efficacy of osimertinib as a second-line treatment may be more limited in patients with BM than those without non-BM lesions. In addition, the effectiveness of osimertinib in patients that had developed initial BM before osimertinib administration was more limited than in the patients that had developed initial BM after acquiring osimertinib resistance.

However, the reasons for the difference in the efficacy of osimertinib in patients with $\mathrm{BM}$ and non-BM are not yet elucidated. We speculate that the possible reasons might be as follows. First of all, a previous study showed that although the concentration of osimertinib is significantly higher than those of other drugs in CSF, it is still significantly lower than that in the plasma [15]. In addition, the tumor microenvironment of BM lesions may be changed compared with the status of initial lesion, resulting in a poorer effect on BM. For example, unlike in non-BM lesions, BM lesions acquired anti-oxidant gene mutations related to cellular stress response, including Keap-1, Nrf2, and P300, which are key factors for the promotion of secondary metastasis $[16,17]$.

Since the ability of osimertinib to control BM in patients with advanced EGFR T790M mutations after obtaining first-generation EGFR-TKI resistance may be more limited, than in those with non-BM lesions, a further improvement of the treatment strategy is highly required. First of all, reducing the incidence of BM in patients with a T790M mutation after obtaining firstgeneration EGFR-TKI resistance is a feasible strategy to improve the survival time. Our previous studies also revealed that first-generation EGFR-TKI combined with chemotherapy as the first-line treatment reduced the incidence of $\mathrm{BM}$ and prolonged the time of $\mathrm{BM}$ $[18,19]$. Furthermore, previous investigations have shown that the combination of RT and first-generation EGFR-TKI have better efficacy than the combination of EGFR-TKI or RT alone [10, 20, 21]. Our previous research also revealed that EGFR-TKI administration after 4 weeks of radiotherapy was more effective [12]. Radiotherapy could not only open the BBB to increase the concentration of intracranial drugs, but also locally control BM [22]. The potential similarity in the effects of osimertinib and RT has not been examined before. In this study, we found that osimertinib combined with radiotherapy in BM patients may lead to better survival benefits than osimertinib alone. However, the time relationship between osimertinib and radiotherapy still needs further exploration in future research. Therefore, 


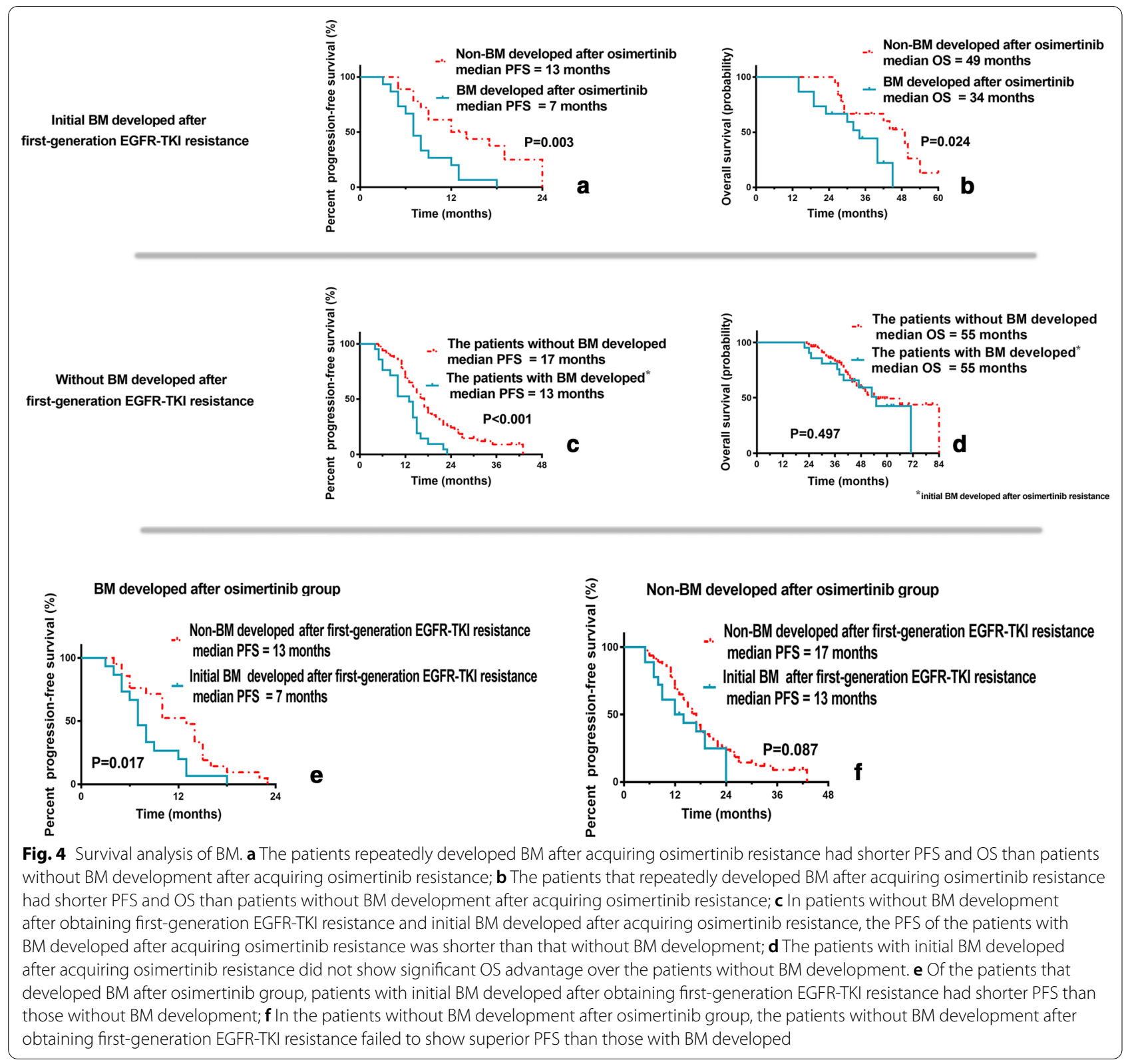

for patients without $\mathrm{BM}$, osimertinib alone may provide good survival benefits. But for BM patients, osimertinib combined with RT may be a better treatment option.

Our study also demonstrated that occurrence of initial $\mathrm{BM}$ during osimertinib did not affect overall survival for the patients carrying T790M mutation but without BM. Although the underlying mechanism still unclear, we speculated that the difference in treatment time, treatment options (different chemotherapy regimens, osimertinib, radiotherapy, and so on), and treatment sequence after BM progression might affect OS. In addition, the previous studies had established a BM preclinical model based on a multi-organ microfluidic chip, and revealed the potential mechanism of obtaining drug resistance in $\mathrm{BM}$, providing a new strategy for overcoming the treatment resistance of lung cancer BM [23, 24]. Therefore, we could find new effective targets for first generation EGFR-TKI or osimertinib-resistant cells through chip model and select the most effective treatment strategy.

The significance and of our research lies in the fact that relatively novel findings have been reported that can reflect the current status and development of medical practice. However, our research still has some limitations. First, this is a non-random retrospective and 
single-institution study that includes unrecognized biases and confounding factors. Second, the sample size is relatively small.

\section{Conclusion}

Our research findings reveal that the efficacy of osimertinib alone as the second-line treatment for control of BM in advanced patients with EGFR T790M mutation after obtaining first-generation EGFR-TKI resistance may be more limited than in non-BM cases. The efficacy of osimertinib in the patients that had developed initial BM before osimertinib administration was more limited than in the patients that had developed initial BM after acquiring osimertinib resistance. Importantly, osimertinib combined with brain RT may exert survival benefits in BM patients.

\footnotetext{
Abbreviations

EGFR: Epidermal growth factor receptor; TKI: Tyrosine kinase inhibitor; NSCLC: Non-small cell lung cancer; BBB: Blood-brain barrier; BM: Brain metastasis; RT: Radiotherapy; CT: Computed tomography; MRI: Magnetic resonance imaging; ECOG: Eastern Cooperative Oncology Group; PS: Performance status; DS-GPA: Disease-specific graded prognosis assessment; WBRT: Whole-brain radiation therapy; SRS: Stereotactic radiosurgery; ctDNA: Circulating tumor DNA; ARMS: Amplification system technology; PFS: Progression-free survival; OS: Overall survival.
}

\section{Acknowledgements \\ None.}

\section{Authors' contributions}

Conceptualization of the study was achieved by XYZ, CHL, and JDG. The research methodology was designed by WN and ANX. Formal analysis of the data was conducted by HZ, TQC, and RBZ. Project administration was carried out by $J \mathrm{LX}$ and $J \mathrm{~L}$. The study resources were obtained by XXZ and BZ. Software analysis of data and figures was conducted by YCS and FP. In addition, supervision of the research was conducted by $\mathrm{BH}, \mathrm{XYZ}$ and WN. Writing the manuscript was carried out by $\mathrm{CHL}, \mathrm{ANX}$, and JDG. Review and editing of the manuscript were carried out by $\mathrm{XYZ}$ and $\mathrm{BHH}$. All authors agreed to be accountable for all aspects of the research. All authors read and approved the final manuscript.

\section{Funding}

This study was financially supported by Interdisciplinary Program of Shanghai Jiao Tong University (Grant No. ZH2018QNA67), Nurture projects for basic research of Shanghai Chest Hospital (Grant No. 2018YNJCM05) and Science and Technology Commission of Shanghai Municipality, China (Grant No.18441904700)

\section{Availability of data and materials}

The enrolled patients agreed to use the information only for this study and refused to share it further.

\section{Declarations}

Ethics approval and consent for participation

The study has been approved by the Ethics Committee and the Institutional Review Board, and was carried out in compliance with Helsinki Declaration.

\section{Consent for publication}

All patients agreed that their information was used for research and signed written informed consent before the collection of information.

\section{Competing interests}

The authors state that they have no conflict of interests.

\section{Author details}

${ }^{1}$ Department of Pulmonary, Shanghai Chest Hospital, Shanghai Jiao Tong University, No.241 Huaihai West Road, Xuhui District, Shanghai 200030, China. ${ }^{2}$ Department of Radiation Oncology, Shanghai Chest Hospital, Shanghai Jiao Tong University, No.241 Huaihai West Road, Xuhui District, Shanghai 200030, China.

Received: 13 January 2021 Accepted: 7 May 2021

Published online: 11 May 2021

\section{References}

1. Shao Y, Zhong DS. Histological transformation after acquired resistance to epidermal growth factor tyrosine kinase inhibitors. Int J Clin Oncol. 2018;23(2):235-42.

2. Mok TS, Wu YL, Thongprasert S, et al. Gefitinib or carboplatin-paclitaxel in pulmonary adenocarcinoma. N Engl J Med. 2009;361(10):947-57.

3. Chai CS, Liam CK, Poh ME, et al. Predictors of acquired T790M mutation in patients failing first- or second-generation epidermal growth factor receptor-tyrosine kinase inhibitors. Cancer Manag Res. 2020;12:5439-50.

4. Di Lorenzo R, Ahluwalia MS. Targeted therapy of brain metastases: latest evidence and clinical implications. Ther Adv Med Oncol. 2017;9(12):781-96.

5. Mamon HJ, Yeap BY, Jänne PA, et al. High risk of brain metastases in surgically staged IIIA non-small-cell lung cancer patients treated with surgery, chemotherapy, and radiation. J Clin Oncol. 2005;23(7):1530-7.

6. Li L, Luo S, Lin H, et al. Correlation between EGFR mutation status and the incidence of brain metastases in patients with non-small cell lung cancer. J Thorac Dis. 2017:9(8):2510-20.

7. Nadler E, Espirito JL, Pavilack M, Baidoo B, Fernandes A. Real-world disease burden and outcomes of brain metastases in EGFR mutationpositive non-small-cell lung cancer. Future Oncol (Lond, Engl). 2020;16(22):1575-84

8. MokTS, Wu YL, Ahn MJ, et al. Osimertinib or platinum-pemetrexed in EGFR T790M-positive lung cancer. N Engl J Med. 2017;376(7):629-40.

9. Ezeife DA, Kirk V, Chew DS, et al. Economic analysis of osimertinib in previously untreated EGFR-mutant advanced non-small cell lung cancer in Canada. Lung Cancer (Amsterdam, Netherlands). 2018:125:1-7.

10. Dong K, Liang W, Zhao S, et al. EGFR-TKI plus brain radiotherapy versus EGFR-TKI alone in the management of EGFR-mutated NSCLC patients with brain metastases. Transl Lung Cancer Res. 2019;8(3):268-79.

11. Sperduto PW, Kased N, Roberge D, et al. Summary report on the graded prognostic assessment: an accurate and facile diagnosis-specific tool to estimate survival for patients with brain metastases. J Clin Oncol. 2012;30(4):419-25.

12. Li C, Guo J, Zhao L, et al. Upfront whole brain radiotherapy for multiple brain metastases in patients with EGFR-mutant lung adenocarcinoma. Cancer Manag Res. 2019;11:3433-43.

13. Shetty V, Babu S. Management of CNS metastases in patients with EGFR mutation-positive NSCLC. Indian J Cancer. 2019;56(Supplement):S31-7.

14. Kelly WJ, Shah NJ, Subramaniam DS. Management of brain metastases in epidermal growth factor receptor mutant non-small-cell lung cancer. Front Oncol. 2018:8:208.

15. Song Y, Liu P, Huang Y, Guan Y, Han X, Shi Y. Osimertinib quantitative and gene variation analyses in cerebrospinal fluid and plasma of a non-small cell lung cancer patient with leptomeningeal metastases. Curr Cancer Drug Targets. 2019;19(8):666-73.

16. Aljohani HM, Aittaleb M, Furgason JM, et al. Genetic mutations associated with lung cancer metastasis to the brain. Mutagenesis. 2018;33(2):137-45.

17. Cacho-Díaz B, García-Botello DR, Wegman-Ostrosky T, Reyes-Soto G, OrtizSánchez E, Herrera-Montalvo LA. Tumor microenvironment differences between primary tumor and brain metastases. J Transl Med. 2020;18(1):1.

18. Li C, Zhang B, Guo J, et al. Correction to: epidermal growth factor receptor (EGFR)-tyrosine kinase inhibitors (TKIs) combined with chemotherapy delay brain metastasis in patients with EGFR-mutant lung adenocarcinoma. Target Oncol. 2019;14(4):491. 
19. Chamberlain MC, Baik CS, Gadi VK, Bhatia S, Chow LQ. Systemic therapy of brain metastases: non-small cell lung cancer, breast cancer, and melanoma. Neuro Oncol. 2017;19(1):i1-24.

20. Chen Y, Wei J, Cai J, Liu A. Combination therapy of brain radiotherapy and EGFR-TKIs is more effective than TKIs alone for EGFR-mutant lung adenocarcinoma patients with asymptomatic brain metastasis. BMC Cancer. 2019;19(1):793

21. Chen Y, Yang J, Li X, et al. First-line epidermal growth factor receptor (EGFR)-tyrosine kinase inhibitor alone or with whole-brain radiotherapy for brain metastases in patients with EGFR-mutated lung adenocarcinoma. Cancer Sci. 2016;107(12):1800-5.

22. Tomasini P, Serdjebi C, Khobta N, et al. EGFR and KRAS mutations predict the incidence and outcome of brain metastases in non-small cell lung cancer. Int J Mol Sci. 2016;17(12):2132.
23. Xu M, Wang Y, Duan $W$, et al. Proteomic reveals reasons for acquired drug resistance in lung cancer derived brain metastasis based on a newly established multi-organ microfluidic chip model. Front Bioeng Biotechnol. 2020;8:612091.

24. Liu W, Song J, Du X, et al. AKR1B10 (Aldo-keto reductase family 1 B10) promotes brain metastasis of lung cancer cells in a multi-organ microfluidic chip model. Acta Biomater. 2019;91:195-208.

\section{Publisher's Note}

Springer Nature remains neutral with regard to jurisdictional claims in published maps and institutional affiliations.
Ready to submit your research? Choose BMC and benefit from:

- fast, convenient online submission

- thorough peer review by experienced researchers in your field

- rapid publication on acceptance

- support for research data, including large and complex data types

- gold Open Access which fosters wider collaboration and increased citations

- maximum visibility for your research: over 100M website views per year

At BMC, research is always in progress.

Learn more biomedcentral.com/submissions 